\title{
Acciones positivas y principio de especialidad. Reflexiones desde la teoría de las fuentes
}

\section{Positive actions and the principle of specialty. Reflections from the theory of sources}

\author{
ANDREA PISANESCHI \\ Catedrático de Derecho Constitucional. \\ Università degli Studi di Siena. \\ andrea.pisaneschi@unisi.it
}

DOI: https://doi.org/10.7203/cc.2.21224

Fecha de recepción: 25/06/2021

Fecha de aceptación: 16/09/2021

\section{Resumen}

El artículo reconstruye las acciones positivas basándose en la teoría económica de los bienes escasos y de las medidas compensatorias que el Estado debe establecer en base al principio de igualdad sustancial. Las acciones positivas, además, se han de calificar como lex specialis. Ello permite evitar, desde el punto de vista dogmático, la eventual confrontación entre las acciones positivas y el principio de igualdad formal. Pues ellas, en efecto, disciplinan unos concretos supuestos especiales que no derogan el principio general, por cuanto se aplican los principios que regula las relaciones entre normas generales y normas especiales. La calificación de la acción positiva como norma especial permite además poder determinar su duración. Las mismas, como todas las normas especiales, han de cesar cuando cesan las razones que justificaron su adopción.

\section{Palabras clave}

Acciones positivas; principio de igualdad; bienes escasos; bienes vitales; ley especial; temporalidad.

\section{Abstract}

The article reconstructs the positive actions based on the economic theory of scarce goods and the compensatory measures that the State must introduce, based on the principle of substantial equality. Furthermore, positive actions are qualified as lex specialis. This makes it possible to avoid, from a dogmatic point of view, the possible contrast of positive actions with the principle of formal equality. In fact, they govern a special case and do not derogate from the general principle, in application of the principles governing the relationship between general norms and special norms. The qualification of the positive action as a special rule also allows determining their duration. They, like all special norms, must cease when the reasons that justified their adoption cease.

\section{Keywords}

Positive actions; principle of equality; scarce goods; goods of life; lex specialis; temporariness. 


\section{Acciones positivas. II. Igualdad formal e igualdad material. III. Acciones positivas y "lex specialis". IV. Breves conclusio- nes. Bibliografía.}

\section{Introducción. Justicia y medios de comunicación}

Es sabido que las acciones positivas vieron la luz en los Estados Unidos de Norteamérica y en particular en el período kennedyano, cuando en 1961 el Presidente Kennedy dictó la Executive Order 10925, que inauguró, precisamente, la era de las affirmative action policy (Jones, 1985; Chang, 1991: 790 y ss.; Drake y Holsworth, 1996). Desde entonces, las acciones positivas se han difundido ampliamente en Europa, en América Latina y en general en todo el mundo, y se utilizan para corregir varias hipótesis de situaciones históricamente discriminadas o para igualar (o reequilibrar) posiciones de desventaja (Drake y Holsworth, 1996; Harris, 1996: 326).

Se definen de formas diversas pero en general puede decirse, adaptando la terminología desde la perspectiva de la regulación económica, que una acción positiva consiste en la atribución de un bien escaso a un grupo humano que históricamente ha tenido un acceso restringido o limitado a ese determinado bien (Gianformaggio, 1988: 33 y ss.).

El concepto de bien puede entenderse en el sentido más variado (desde los bienes de naturaleza económica a bienes conexos con situaciones jurídicas subjetivas y a derechos de diversos tipos, hasta incluso los bienes inmateriales como por ejemplo el tiempo, ejemplo clásico de las acciones positivas acordadas en favor de los trabajadores y de las trabajadoras padres o madres). Veremos después que la definición más coherente es probablemente aquella procesalista de bien vital (bene de la vita) que resume sintéticamente una amplia variedad de situaciones jurídicas subjetivas.

El concepto de lo "escaso" también es, a su vez, muy variable. Es escaso un bien que por naturaleza es finito, pero es escaso también un bien que la normativa de aplicación lo considera de acceso limitado (la representación política, la representación en los Consejos de administración de las sociedades o el mismo acceso al proceso que la ley solo considera posible cuando concurren determinadas situaciones subjetivas). El concepto de escasez es, en cualquier caso, un concepto relativo, necesariamente ligado al momento histórico, al país y al concreto grupo de que se trate (Conrad, 1995: 5 y ss.; Goldman, 1979: 141 y ss.; Verkerke, 1989: 1499).

Existen bienes que pueden ser escasos en un determinado país y en absoluto sean escasos en otros. Los grupos humanos pueden a su vez ser de distinta naturaleza. Puede tratarse de un grupo determinado por el género (el caso clásico es la diversidad hombre-mujer) o de raza, o un grupo étnico, o de minoría lingüística. Lo que ello viene a demostrar es que por una parte la Constitución determina un concreto grupo como merecedor de protección, ya lo sea de modo general o incluso indirecta. A la determinación constitucional subyace una valoración preliminar sobre la situación histórica de desventaja, merecedora por ello de una intervención redistributiva. Por ejemplo, la Constitución italiana en el art. 3 dispone que "todos los ciudadanos tienen la misma dignidad social y son iguales ante la ley sin distinción de sexo, raza, lengua, religión, opinión 
política o condiciones personales o sociales", refiriéndose con ello, implícitamente, a diferentes grupos humanos históricamente discriminados.

Por estas respectivas características la acción positiva parece plantear una tensión y hasta un conflicto entre las dos dimensiones de la igualdad que generalmente suelen contemplarse en las constituciones contemporáneas: la dimensión formal y la material. Mientras el aspecto formal postula la igualdad de los ciudadanos ante la ley, en el sentido de que las diferencias no pueden constituir un motivo de tratamiento normativo diferente, la igualdad material impulsa a la ley a realizar un trato diferente para poder lograr un determinado objetivo (Rosenfield, 1991: 5 y ss.; Livingston, 1979).

En tanto que la igualdad formal excluye la diferencia en el tratamiento por razones de sexo, raza, religión, etc., la igualdad material toma como punto de partida el propio sexo, la raza o la religión para determinar el tratamiento jurídico de los individuos y sus grupos y, por consiguiente, de su concreta posición social. Se trata, en el fondo, de la paradoja advertida por el juez Scalia en el famoso voto particular a la sentencia Johnson v. Transportation Agency: mediante una intervención de soporte a los grupos débiles, la garantía para que la raza y el sexo deban ser jurídicamente indiferentes, se convierte en su opuesto. Y, por lo demás, es por esta misma razón que en la literatura americana -por parte de los críticos de las acciones positivas- dichas acciones hayan sido definidas como discriminación inversa ("reverse discrimination") con el fin de sostener que tales acciones se fundamentan en una discriminación, esto es, en la violación del principio de igualdad.

La expresión "reverse discrimination" que, por lo demás, ha trascendido al lenguaje común para indicar sintéticamente las acciones positivas, es merecedora de alguna reflexión, que después haremos, precisamente en la medida en que a la misma subyace una distinción-contraposición entre la igualdad formal y la igualdad material, contraposición que, sin embargo, por las razones que se verán, no es tan fuerte como se quisiera (Ballestrero, 1988; Amedeo y Dolso, 1988: 1221 y ss.). Otra cuestión es, no obstante, sostener que los dos conceptos -igualdad formal e igualdad material- deban considerarse diferentes. Pues ellos, de hecho, tienen una diversa matriz histórica y una diferente funcionalidad; además de que se aplican con diferentes modalidades (Rosenfield, 1991: 15).

Bajo el primer punto de vista, en efecto, la igualdad formal postula una visión, por así decir, estática de la igualdad, coherente con una forma de Estado de tipo liberal, no intervencionista, socialmente homogénea o por lo menos considerada tal desde el punto de vista de la relevancia jurídica. Por el contrario, la igualdad material postula una visión dinámica de la igualdad que se corresponde con una forma de Estado de tipo social, fuertemente intervencionista y socialmente muy heterogénea. En la igualdad sustancial se halla, además, implícita una finalidad: en efecto, es función del Estado remover algunas desigualdades concretas.

Bajo el segundo punto de vista de la igualdad formal se deriva la prohibición de discriminaciones directas e indirectas. Son discriminaciones directas aqueIlas situaciones en las que una norma excluye expresamente a una categoría de sujetos del disfrute de un derecho o que les somete a un tratamiento inferior. La discriminación indirecta, en cambio, se manifiesta siempre que la norma para el disfrute de un derecho o el ejercicio de una facultad establezca la concurrencia de determinados requisitos con lo que, indirectamente, se excluye de la disposición de aquellos derechos a una determinada categoría de personas que no se halla dotada de tales requisitos exigidos. Piénsese en una ley que establezca para el acceso a los cuerpos de bomberos una determinada altura mínima sin que haya diferencias en base al sexo, pero discriminando con ello, obviamente, al sexo femenino que se halla caracterizado por alturas medias 
inferiores a las previstas por la norma (la ley fue declarada inconstitucional por el Tribunal Constitucional italiano por sentencia núm. 163, de 1993) (Ainis, 1993: 891 y ss.; Anzon, 1993: 901 y ss.).

Es decir, que la igualdad formal desarrolla la función de sancionar un comportamiento legislativo, directo o indirecto, que tiene naturaleza de exclusión. Por el contrario, la igualdad sustancial desarrolla la función diferente de prescribir una determinada acción con el fin opuesto de llevar a cabo una inclusión que el ordenamiento no ha sido capaz de efectuar sin más. Desde una perspectiva dogmática puede, por tanto, incluso decirse que la igualdad formal tiene una naturaleza esencialmente descriptiva porque impone al legislador un "no hacer" (no realizar discriminaciones ni directas ni indirectas) mientras que la igualdad sustancial tiene por el contrario una naturaleza prescriptiva porque impone un "hacer" determinado al legislador (Caravita di Toritto, 1984; Ainis, 1992: 582 y ss.).

También son normas funcionalmente distintas: la igualdad formal promueve la no discriminación; la igualdad sustancial atribuye la utilización de un bien, un derecho o una facultad, a quien con relación a aquel bien, derecho o facultad quedó históricamente excluido. Pero ambas tienden a aplicar la igualdad en las dos dimensiones de no discriminación y de inclusión.

\section{Igualdad formal e igualdad material}

Sentado, pues, que la igualdad formal y la material se refieren a supuestos ontológicamente diversos, es oportuno realizar alguna precisión sobre las diversas modalidades de aplicación de los dos principios.

Ya quedó dicho que la igualdad formal se manifiesta a través de medidas negativas (prohibiciones, proscripción de discriminaciones) mientras que la igualdad sustancial a través de intervenciones positivas (incentivos, compensaciones...) (Jori, 1977: 405; Lumia, 1978: 431 y ss.; Gavazzi, 1983: 37 y ss.). Por consiguiente, la primera se basa en un esquema tradicional -típico del Estado decimonónico y de la concepción autoritaria del derecho- mandato-sanción, mientras que la segunda se basa en un modelo bastante diferente definido como de "sanciones positivas" (Bobbio, 1971: 237; Lombardi, 1976: 98 y ss.). La sanción positiva, debiendo salir al paso de una inaplicación del ordenamiento en cuanto al disfrute de un bien, derecho o facultad, tiene por objeto su atribución a una categoría desventajada de aquel bien, derecho o facultad. Tratándose de una exigencia o, incluso de un conjunto de exigencias, tal como ya se ha afirmado, puede usarse la definición normalmente utilizada en el ámbito del proceso "de bienes vitales". El bien de la vida representa simplemente aquel conjunto de relaciones jurídicas que el actor solicita en el ámbito de la estructura procesal. El bien vital en este caso concreto es aquel bien escaso al que tiene un limitado acceso un determinado grupo social.

Y no es menos significativo que en concreto ese bien vital pueda presentar importantes variables. Piénsese en las llamadas cuotas electorales en las que la acción positiva puede ser entendida como una posibilidad que el ordenamiento atribuye a un determinado grupo que se considera históricamente en desventaja para obtener un resultado determinado, o bien que se considere como un propio y verdadero "derecho al resultado" que se reconoce para aquel grupo concreto.

En el primer sentido se orientó en el pasado por ejemplo el Tribunal Constitucional italiano que en una sentencia de 1995 (la núm. 422) afirmó que las acciones positivas en favor de sujetos desventajados en materia electoral, no podían producir un resultado determinado, teniendo que limitarse en cambio a disponer de los medios para que tales sujetos que se hallaban en desventaja 
pudieran lograr aquel resultado. En otras palabras, la ley no habría podido establecer una reserva de puestos sino solo una reserva de candidaturas para poder proporcionar a todos la paridad de chances. La pertenencia a un sexo u otro no podía por lo tanto convertirse en un requisito para la elección. La persistencia de la infrarrepresentación femenina en materia electoral ha ido, sin embargo, conduciendo con el tiempo al Alto Tribunal italiano a precisar dicha orientación en el sentido de que -en presencia de una previsión constitucional que hace del equilibrio en la representación una verdadera y propia obligación- las leyes que imponen la presencia de ambos sexos en las listas electorales no solo son admisibles, sino que además han de ser tales que puedan realizar dicho equilibrio sancionado por la Constitución' (Brunelli, 2002: 615).

En el segundo sentido (es decir, si se concibe como derecho a un resultado y no simplemente como una posibilidad) se ha orientado mucha legislación de carácter electoral en América Latina y en América central. En México por ejemplo, se halla prevista una acción positiva indígena interpretada como una vinculación muy fuerte para los partidos, los cuales son obligados a presentar solo candidaturas indígenas en los distritos donde concurra una concentración indígena que supere el 60\% de la población total (Bindi, Groppi y Pisaneschi, 2018: 215 y ss.).

Volviendo a los principios generales, aun cuando se habla de incumplimiento -que generalmente supone un comportamiento culpable- el incumplimiento que justifica la acción positiva no es necesariamente un incumplimiento imputable (es decir, como consecuencia de una culpa) porque puede depender -y normalmente depende- de variadas circunstancias, históricas, sociales, culturales... Ello no obstante, frente a una previsión constitucional de inclusión, cuando se debe recurrir a la acción positiva esta representa un efecto implícito de que el ordenamiento jurídico no ha sabido poner remedio a tales violaciones constitucionales de las que se acaba derivando en la "sanción" de la acción positiva. Esta, por tanto, no diversamente a cuanto significa la sanción, se configura como extrema ratio cuando las medidas generales de incentivo social ordinario, que el Estado haya puesto en marcha para remover la desigualdad, no han producido el resultado esperado.

En todo caso, de ello deriva una primera diferencia fundamental entre la igualdad formal y la material que se refleja en el objeto de las acciones positivas (Bobbio, 1978). Mientras la igualdad formal es una igualdad abstracta en el punto de partida, que tutela un derecho preexistente ya en toda persona (el derecho a no ser discriminado negativamente), la igualdad sustancial, y por consiguiente la acción positiva, atribuye un "bien de la vida" (o bien jurídicamente protegido) a quienes se hallan privados de tal bien, realizando así la igualdad en el punto de llegada (o de resultado) y no en el punto de partida. Desde el punto de vista civilista la acción positiva podría, por tanto, ser asimilada no a una obligación de proporcionar medios sino a una obligación de lograr un resultado. Existe, además, una segunda diferencia entre los instrumentos para realizar la igualdad formal y las acciones positivas: la igualdad formal tiene por objeto al individuo aislado, mientras que, por el contrario, la acción positiva tiene por objeto al grupo.

Baste pensar en el clásico íncipit típico de la definición de igualdad formal ("todos los ciudadanos son iguales ante la ley") del que se deriva claramente que el objeto de la norma son los sujetos individuales. Ciertamente, ello no impide que dicha norma pueda ser aplicada también a grupos de ciudadanos pero, al tiempo, no hay duda de que la misma va prioritariamente destinada a

1 Corte costituzionale, sentencia núm. 49 de 2003. 
los individuos en particular. Por el contrario, la acción positiva se dirige exclusivamente a la protección de un grupo social conformado por una determinada característica que, por razones históricas, culturales, sociales, etc., es suficiente para atribuir a dicho grupo una unidad identitaria, cuyo valor es constitucionalmente reconocido. Importa también destacar que la acción se dirige a un grupo concreto y determinado y no, en hipótesis, a varios grupos reunidos por el hecho de hallarse en desventaja. De hecho, la acción positiva es necesariamente selectiva y ello la distingue por ejemplo de las medidas generales de incentivo, de promoción, de desarrollo, etc., que no se refieren a un grupo específico sino al contrario, a diversos grupos sociales.

$\mathrm{Si}$, por lo tanto, el beneficiario de la acción positiva es un grupo mientras que el beneficiario de la igualdad formal es el particular, también las acciones necesarias para atribuir las dos igualdades diversas son bastante diferentes. Las acciones para la defensa de la igualdad formal se basan, de hecho, en la generalidad y la abstracción y tienden a producir principios unificadores. Ellas están fundadas en situaciones abstractas de la "ley igual para todos" y es en la generalidad y abstracción de la ley donde reside efectivamente, la garantía mínima de la no discriminación. Si la norma no se dirige a una persona particular, sino a una generalidad de personas, y además es abstractamente repetible, cada uno queda garantizado por un tratamiento igual y previsible (Cartabia, 1996: 65).

Por el contrario, en la acción positiva la norma tiene un nivel de generalidad y abstracción atenuado porque la misma se dirige a un grupo social determinado. No es, por tanto, general en su esencia pues no regula hechos o comportamientos sin referencia a situaciones o sujetos determinados; pero no tiene tampoco naturaleza provisional puesto que se dirige, no a una persona determinada, sino a un grupo social entendido como ente exponencial (un grupo étnico, un grupo religioso, etc.) y no como conjunto o suma de individuos particulares. En efecto, el grupo es variable pudiendo ampliarse o reducirse con el tiempo siendo ello jurídicamente irrelevante ya que la acción positiva no tutela a las personas aisladas que componen el grupo sino al grupo en sí mismo considerado (Romano, 1945). También la abstracción, entendida como la repetibilidad de la regla durante un tiempo indeterminado, es limitada (Crisafulli, 1935; Bobbio, 1958). Tal como veremos, la acción positiva se ejerce en un tiempo determinado. Aun cuando no se trate de una hipótesis de sunset legislation, (según el modelo americano) en la que algunas leyes tienen un plazo de caducidad previsto ya en la misma ley, las acciones positivas se hallan fisiológicamente caracterizadas por un plazo fijado en torno al logro del objetivo que se pretende.

No es, ciertamente, un fenómeno nuevo ni típico exclusivamente de las acciones positivas: la pérdida de las características propias de las normas jurídicas y la existencia de zonas grises de frontera entre lo que es normativo y lo que no lo es deriva de las características de la sociedad actual, fragmentada en grupos sociales diferentes que necesitan de tratamientos a su vez diferenciados. Por lo demás, el procedimiento de producción del derecho se halla hoy en día fuertemente contratualizado. Intervienen en él sujetos portadores de intereses particulares (desde los sindicatos a las organizaciones profesionales, o a los grupos de presión, etc.) con la consecuencia de que el resultado normativo a menudo se halla caracterizado más por elementos de particularidades que por la "histórica" generalidad y abstracción.

Por consiguiente, igualdad formal e igualdad sustancial delimitan tipos distintos; los instrumentos para realizar la igualdad sustancial son diferentes y en algunos casos opuestos (baste pensar en el tema de la generalidad y la abstracción); las acciones positivas, en la medida en que atribuyen un bien de la vida "escaso", constituyen un -aunque no solo- instrumento para realizar 
la igualdad sustancial. Y ello es así porque, mientras la igualdad formal tiene naturaleza meramente descriptiva abstracta, la igualdad sustancial tiene una naturaleza prescriptiva pero, además, intrínsecamente valorativa. Es la diferencia que Dworkin subrayaba entre la igualdad que considera a los hombres como "equal" (es decir, como si fuesen iguales), respecto de la igualdad que los trata "equally" (Dworkin, 1982: 297; Bobbio, 1978: 18 y ss.).

Llegados a este punto se nos plantea inevitablemente una cuestión. Siempre puede haber conflictos entre diversas normas, siempre pueden surgir conflictos entre derechos constitucionalmente protegidos, aun cuando la Constitución los reconozca sin conflicto entre ellos. El conflicto, por tanto, no se refiere exclusivamente a la posición activa respecto de la posición pasiva de un derecho según el esquema de facultad-obligación, o de poder-subordinación. Sino que se refiere a posiciones activas constitucionalmente tuteladas. Sería posible por lo tanto un conflicto entre el principio de igualdad formal y el principio de igualdad material; un conflicto entre el principio que proclama que todos los ciudadanos son iguales ante la ley y la posibilidad de tratar diferenciadamente mediante la atribución de un bien jurídicamente protegido, y determinado, a algunos grupos sociales. Este conflicto normalmente se resuelve a través de la técnica del balance con una suerte de prius valorativo en favor de la igualdad material.

Con la igualdad material, se dice, la Constitución lleva a cabo una opción valorativa seleccionando expresa y apriorísticamente las posiciones más débiles merecedoras de tutela. Esta opción es condicionante precisamente porque la opción valorativa que se lleva a cabo selecciona, destacándola hacia arriba, determinadas relaciones a disciplinar y regular caracterizando, por lo demás, la misma forma de Estado. Si el derecho por definición siempre constituye una alteración del estado de naturaleza, la igualdad material constituye una alteración más fuerte aun al establecerse en ventaja de concretas situaciones subjetivas cuyo valor a tutelar se considera prioritario. De ello deriva, por consiguiente, que la acción positiva, como aplicación del principio de igualdad material, es a la vez expresión de una norma constitucional propositiva y valorativa que no podría dejar de prevalecer (siempre que se adopte con pleno respeto de las reglas necesarias) en el balance que confronte la igualdad formal con la material (Ainis, 1999: 359).

\section{Acciones positivas y "lex specialis"}

En realidad, a resultados análogos puede llegarse también a través de un razonamiento menos neoconstitucionalista, menos relativista ( $y$, por lo tanto, menos opinable e intrínsecamente político) y más atento en cambio a las características formales de las normas que contienen la acción positiva.

En efecto, se ha sostenido hasta ahora que la igualdad material regula unos supuestos diferentes a los de la igualdad formal aun persiguiendo el mismo fin (la aplicación de la igualdad). En particular, determina situaciones específicas merecedoras de intervención redistributiva de cuya aplicación se hace cargo la acción positiva. La igualdad formal, en cambio, determina la regla general de no discriminación. Encuadrada en estos términos la relación entre la igualdad formal y la igualdad material no puede, sin embargo, más que llevarnos a la conclusión de que la igualdad formal constituye la regla general mientras que la igualdad material -y por tanto la acción positiva- es la excepción. Y, por lo demás, también en esto la literatura es concorde. Las concreciones para las cuales se requiere la intervención positiva se hallan determinadas ya sea 
subjetivamente (como grupos concretos), ya sea objetivamente a través de la atribución de aquel bien vital que el Estado debe atribuir para reequilibrar las posiciones.

Pero, de ser así, no parece ilógico configurar la relación entre la igualdad formal y la igualdad material como una relación de genus y species. La igualdad formal constituiría el genus mientras que la igualdad material sería la species. La primera es norma general mientras la segunda es norma especial. Por consiguiente, la acción positiva en la medida en que atribuye mediante un "hacer" un determinado bien vital a un determinado grupo social, no es en realidad sino una ley especial, mientras que la normativa que en ese concreto ámbito aplica el principio negativo de no discriminación configura la norma general.

Si se realiza esta construcción y se la comparte, el primer efecto, bastante relevante desde el punto de vista teórico, es que no pueda haber conflicto entre igualdad formal e igualdad material, del mismo modo que no se plantea un conflicto jurídico entre la ley general y la especial. Pues se trata de ámbitos o áreas materiales con regulación distinta.

Los dos ámbitos no interfieren uno en otro, como por lo demás se expresaba claramente en el derecho romano cuando se afirmaba que "lex specialis per generalia non derogatur", principio en el que se pone de relieve implícitamente el diferente ámbito material regulado por las normas y por consiguiente la no superposición ni tampoco la posible contraposición entre ellas. La ley especial, en la teoría de las fuentes, aun estableciendo reglas deferentes a las de la ley general, no genera una antinomia -por ejemplo una derogación- con la ley general precisamente porque los ámbitos materiales que se regulan son diferentes (Gavazzi, 1959; Bobbio, 1967).

Es cierto que la ley especial debe encontrar un fundamento justificativo "fuerte" que le permita llevar a cabo esa función de "legítima derogación" en relación con los principios generales. Pero queriendo prescindir de los principios constitucionales, y acogiéndose una vez más a la teoría de las fuentes, también es sabido que las normas especiales se someten a varias limitaciones. El primer límite, generalmente compartido y a menudo incluso codificado, se sustancia en el principio de que las leyes especiales no se aplican más allá de los casos y los tiempos a que ellas mismas se someten precisamente en cuanto que son especiales respecto de la normativa que se considera general. Este principio como se sabe implica dos efectos.

El primero es que las leyes especiales son normalmente leyes temporales. En la medida en que derogan un principio general para un fin predeterminado, el objetivo es lograr esa finalidad y, por lo tanto, no tienen por qué tener una duración indeterminada. La especialidad produce inevitablemente una limitación temporal que va conectada al logro del objetivo que la propia ley se propone alcanzar y en función del cual se justifica la derogación de la regla general.

El segundo es la prohibición de la interpretación analógica. Si la norma es especial, lo es en razón del concreto supuesto de dicha regla, pues solo ella es merecedora del tratamiento diferenciado. De tal modo que sería una contradicción lógica evidente que se extendiera analógicamente a otros supuestos una norma que es especial. Se observa que estos dos primeros requisitos se aplican perfectamente a las acciones positivas. Siempre se ha dicho que las acciones positivas no pueden tener una duración indeterminada y para corroborarlo se recurre normalmente a principios sociológicos, económicos, politológicos, etc. Lo cierto es que, desde un punto de vista estrictamente jurídico, las acciones positivas son por su propia naturaleza fisiológicamente temporales, pues, de otro modo, simplemente perderían el carácter de especialidad. Pero entonces sí que se plantearía un conflicto irresoluble con el principio de igualdad formal. 
También la prohibición de interpretación analógica resulta perfectamente coherente con la naturaleza de las acciones positivas. La acción positiva, en efecto, se halla estrechamente vinculada a la tutela de los supuestos previstos en la constitución, previamente seleccionados y que el legislador decide, en determinados ámbitos, tutelar con normas especiales. La normativa en este caso no falta por definición, porque existe la regla general que es el principio de igualdad formal. En ausencia de una específica acción positiva, por lo tanto, simplemente deberá aplicarse el principio negativo de no discriminación, dado que evidentemente el legislador no ha considerado necesario adoptar la "sanción positiva" especial.

La normativa especial, además, en cuanto es derogatoria del derecho ordinario, desde el punto de vista del control de la constitucionalidad, debe ser ampliamente justificada por el legislador (tanto es así que precisamente respeto de las leyes especiales se ha sostenido la admisibilidad del vicio de exceso de poder legislativo como contradicción entre los fines declarados y los fines logrados) y sometida a un riguroso control de razonabilidad. La motivación constituye la razón de la intervención especial: justamente es en este momento cuando entran en juego factores sociológicos, económicos, históricos, etc., que legitiman la acción positiva. Tales factores constituyen la esencia de la motivación de la norma especial y sirven para establecer los límites de la motivación. No redundan, como a veces puede parecer en la literatura sociológica, en genérico primado de valores respecto de la igualdad formal, cosa que aun supondría un contraste entre igualdad formal e igualdad material. Pero ellos son fundamentales también para consentir el control jurisdiccional de la opción escogida según los tradicionales principios de control lógico-jurídico de la motivación (contradicción, falta de lógica, incoherencia).

También el control sobre la racionabilidad y sobre la proporcionalidad de la medida es necesario y debe basarse en cánones consolidados acerca de la necesaria coherencia entre situación de hecho y previsión normativa y sobre el ya consolidado test de proporcionalidad.

\section{Breves conclusiones}

Evidentemente la reconstrucción de la acción positiva en el ámbito del principio de especialidad tiene, además, la función de poder determinar cierta forma de jerarquía entre la igualdad formal y la igualdad sustancial. La atribución del bien de la vida - o del bien escaso- a un determinado grupo social implica la sustracción de algunos casos al principio de igualdad formal. Y ello, como se ha dicho, es absolutamente legítimo cuando concurren algunas circunstancias, de naturaleza sustancial, que limitan el principio de igualdad. No en vano la acción positiva es lex specialis legitimada precisamente por la existencia de un principio general, cual es la igualdad, pero suspendido en relación a un determinado supuesto hasta su reposición cuando hayan cesado las exigencias que justificaron la acción positiva.

Es así como puede superarse la paradoja del juez Scalia acerca del hecho de que, a través de la intervención de sostén a los grupos débiles, la garantía de que la raza y el sexo deban permanecer jurídicamente indiferentes se convierte en su propio opuesto ("reverse discrimination" o discriminación inversa). 
Ainis, M. (1992). Azioni positive e principio di eguaglianza. Giurisprudenza costituzionale, 582-608.

Ainis, M. (1993). L'eccezione e la sua regola. Giurisprudenza costituzionale, 891-896.

Ainis, M. (1999). Cinque regole per le azioni positive. Quaderni costituzionali, 359-372.

Amedeo, S. y Dolso, G. P. (1988). La Corte costituzionale e le discriminazioni alla rovescia. Giurisprudenza costituzionale, 1221-1248.

Anzon, A. (1993). L'additiva di principio nei giudizi in via principale. Giurisprudenza costituzionale, 891-911.

Ballestrero, M. V. (1988). Azioni positive e discriminazioni alla rovescia. Una importante sentenza della Corte Suprema degli Stati Uniti. Lavoro e diritto, 1988, 53-78.

Bindi, E.; Groppi, T.; y Pisaneschi, A. (2018). La democracia a juicio. Estudio comparativo de la jurisprudencia del TEPJF en el proceso electoral 2017-2018. Pisa: Pacini.

Bobbio, N. (1958). Teoria della norma giuridica. Torino: Giappichelli.

Bobbio, N. (1967). Sui criteri per risolvere le antinomie. Studi in onore di Antonio Segni. Milano.

Bobbio, N. (1971). Sulle sanzioni positive. En Scritti dedicati ad Antonio Raselli (pp. 229-249). Milano: Giuffrè.

Bobbio, N. (1978). Eguaglianza ed egualitarismo. En AAVV, Eguaglianza e egualitarismo. Roma: Armando.

Brunelli, G. (2002). Un "ombrello" costituzionale per le azioni positive elettorali. Quaderni costituzionali, 3, 615-617.

Caravita di Toritto, B. (1984). Oltre l'eguaglianza formale: un'analisi dell'art. 3 comma 2 della Costituzione, Padova: Ist. dir. pubbl. Fac. giur.-Univ. Roma.

Cartabia, M. (1996). Le azioni positive come strumento del pluralismo? En R. Bin y C. Pinelli (eds.), I soggetti del pluralismo nella giurisprudenza costituzionale, Torino: Giappichelli.

Chang, D. (1991) Discriminatory Impact, Affirmative Action and Innocent Victims: Judicial Conservatism or Conservative justice? Columbia Law Review, 91, 790-844. Disponible en: https://digitalcommons.nyls.edu/cgi/viewcontent. cgi?referer=https://www.google.com/\&httpsredir=1\&article=1306\&context $=$ fac articles chapters

Conrad, C. A. (1995). The economic cost of affirmative action: a review of the evidence. En M. C. Simms (ed.), Economic perspectives on affirmative action (pp. 31-54), Washington: University Press of America.

Crisafulli, V. (1935). Sulla teoria della norma giuridica. Roma: Anonima Romana Editoriale.

Drake, W. A., y Holsworth, R. D. (1996). Affirmative Action and the Stalled Quest for Black Progress. Chicago: University of Illinois Press.

Dworkin, R. (1982). I diritti presi sul serio. Bologna: II Mulino.

Gavazzi, G. (1959). Delle antinomie. Torino: Giappichelli.

Gavazzi, G. (1983). Diritto premiale e diritto promozionale. En AAVV, Diritto premiale e sistema penale. Milano: Giuffrè.

Gianformaggio, L. (1988). Eguaglianza, donne, diritto. Bologna: II Mulino.

Goldman, A. H. (1979). Justice and reverse discrimination, Princeton: Princeton University Press

Harris, L. (1996). The future of Affirmative Action. En G. E. Curry (ed.), The affirmative Action debate. Reading: Basic Books.

Jones, J. (1985). Equality and Prohibition of Discrimination in Employment: USA. 
Bulletin of Comparative Labour Relations, 14, 207-231. Disponible en: https:// repository.law.wisc.edu/s/uwlaw/media/22805

Jori, M. (1977). Esiste una funzione promozionale del diritto. Dir. Soc. 1977, 405-419. Livingston, J. C. (1979). Fair game? Inequality and affirmative actions. San Francisco: W. H. Freeman and Company.

Lombardi, G. (1976). Funzione promozionale del diritto, Pubblica Amministrazione e sanzioni positive. Amministrare, 1, 98 y ss.

Lumia, G. (1978). A proposito di struttura, funzione e ideologia nel diritto. Sociologia del Diritto, 2, 431-439.

Romano, S. (1945). L'ordinamento giuridico, Firenze: Sansoni.

Rosenfield, M. (1991). Affirmative action and justice. A philosophical and Constitutional inquiry. New Haven-London: Yale University Press.

Verkerke, J. H. (1989). Compensating victims of preferential employment discrimination remedies. The Yale Law Journal, 98, 1479-1499. Disponible en: https:// digitalcommons.law.yale.edu/cgi/viewcontent.cgi?article=7221\&context=ylj 